\section{Scleral contact lenses for overnight wear in the management of ocular surface disorders}

M.J. TAPPIN, K.W. PULLUM, R.J. BUCKLEY

\begin{abstract}
Purpose To describe the use of overnight wear scleral contact lenses ( $\mathrm{Scl}$ CLs). The authors describe 7 patients using this modality of contact lens wear. Most of the lenses were made from highly gas-permeable materials, but a long-standing case is also reported when the lenses were made from PMMA, which is impermeable to gases.

Background There is a range of therapeutic indications for the use of Scl CLs. The development of rigid gas-permeable (RGP) materials has widened this range.

Case reports Seven case reports are presented which describe patients in whom severe ocular surface disease has been managed with overnight-wear Scl CLs. The indications were: corneal exposure, post-radiotherapy complications, Stevens Johnson disease, recurrent erosion and congenital or postsurgical lid defects.

Conclusion Scl CLs provide a therapeutic option for a range of complicated corneal and ocular surface conditions for which the treatment by other methods is either unsuitable or less effective. They have several advantages over silicone rubber and hydrogel lenses. The relative ease of handling for some patients allows removal for cleaning, their rigidity gives stability and a high degree of protection to the ocular surface, and the presence of a pre-corneal fluid reservoir optically neutralises an irregular corneal surface. Highly oxygen-permeable materials enable consideration of overnight wear in appropriate circumstances.
\end{abstract}

M.J. Tappin

K.W. Pullum

R.J. Buckley

Moorfields Eye Hospital London EC1V 2PD, UK

K.W. Pullum Oxford Eye Hospital Oxford, UK

\section{R.J. Buckley}

Department of Optometry and Visual Science

City University

London, UK

Prof. R.J. Buckley, FRCS, FRCOphth

57A Wimpole Street London W1G 8YP, UK

e-mail: RJBcity@aol.com

Received: 4 May 2000 Accepted in revised form: 19 September 2000
Key words Exposure keratitis, Gas permeable, Overnight wear, Scleral contact lenses, Stevens Johnson disease, Therapeutic

Hydrogel or silicone rubber contact lenses are routinely used to maintain corneal hydration, to protect the cornea from abnormal lashes or lid margins or to assist corneal wound healing. ${ }^{1}$ Overnight wear may be a prerequisite for successful therapeutic contact lens wear as the symptoms during or following sleep may be as bad as, or worse than, during the day.

\section{A distinction between continuous wear and overnight wear}

Therapeutic hydrogel or silicone rubber lenses are usually left in situ continuously for a period of days or weeks without removal. One reason for this is that many patients find removal and reinsertion an intractable problem. It may therefore be the case that continuous wear is unavoidable whether or not overnight wear is required. As a consequence, significant surface spoilation may take place increasing the risk of infection, ${ }^{2}$ and in cases where the eye is sighted, reducing the vision.

Key advantages and disadvantages of scleral contact lenses

One of the main advantages of a scleral contact lens ( $\mathrm{Scl} \mathrm{CL}$ ) is the scleral bearing by which it is held in place and not dislodged by the lids. Scl CLs are often relatively comfortable even for the unadapted eye because the lid margin is in contact with the surface of the lens rather than the edge. An optimally fitted Scl CL entraps a pre-corneal fluid reservoir which provides optical neutralisation of an irregular corneal surface and corneal hydration.

\section{Indications for Scl CLs}

Any eye condition, including some ocular surface diseases, for which contact lens correction is an option, can be considered for Scl CLs if their unique advantages can be applied. ${ }^{3}$

The pre-corneal fluid reservoir entrapped behind a Scl CL may maintain corneal hydration in severe ocular surface diseases such as Stevens Johnson disease, cicatricising pemphigoid and in dry eye conditions of various aetiologies. Scl CLs provide particularly effective protection against trichiasis or lid margin keratinisation for the duration of wear, and there is potential for the encouragement of corneal wound healing in some cases. 


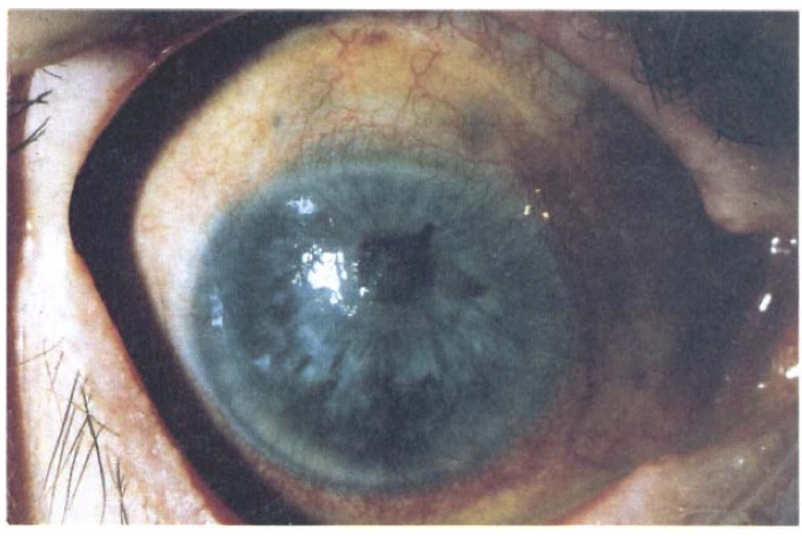

Fig. 1. Case 1. Corneal epithelial erosion giving rise to a marked irregularity of the corneal surface, which is shown by the irregularity of the illuminating light source reflex. The lens is not in situ in this figure.

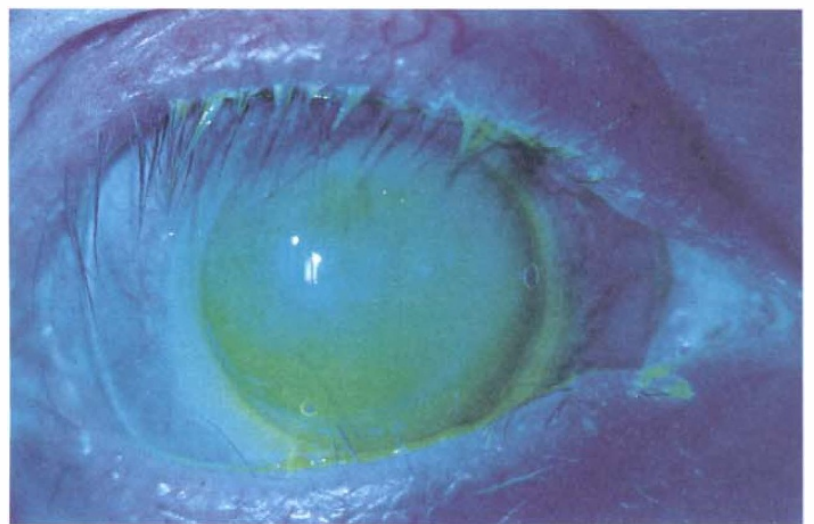

Fig. 3. Case 2. Steven Johnson disease. The first RGP Scl CL issued had full corneal clearance.

It is also possible that the patient, or an assistant, may be able to insert and remove Scl CLs when this cannot be done with hydrogel or silicone rubber lenses. Hence a further therapeutic indication for Scl CLs may be when daytime wear without overnight wear is preferred.

\section{Disadvantages of Scl CLs}

There are widely held misgivings about Scl CLs. The fitting process is comparatively complex, requiring a range of diagnostic lenses and the necessary knowledge to put them to appropriate use. They have traditionally been made in polymethyl methacrylate (PMMA) and cover a large area of the anterior globe, considerably reducing the oxygen available to the cornea.

Fenestrations in scleral lenses can provide some tear exchange to improve corneal oxygenation, but also admit air bubbles to the pre-corneal reservoir, potentially causing visual disturbances and localised dehydration. Fenestrations also cause 'settling back' of the lens on the globe with consequent corneal contact and compression at the limbus. While these problems are surmountable for daily wear only, the greater requirement for therapeutic contact lenses is for both day and overnight wear, so Scl CLs are rarely considered.

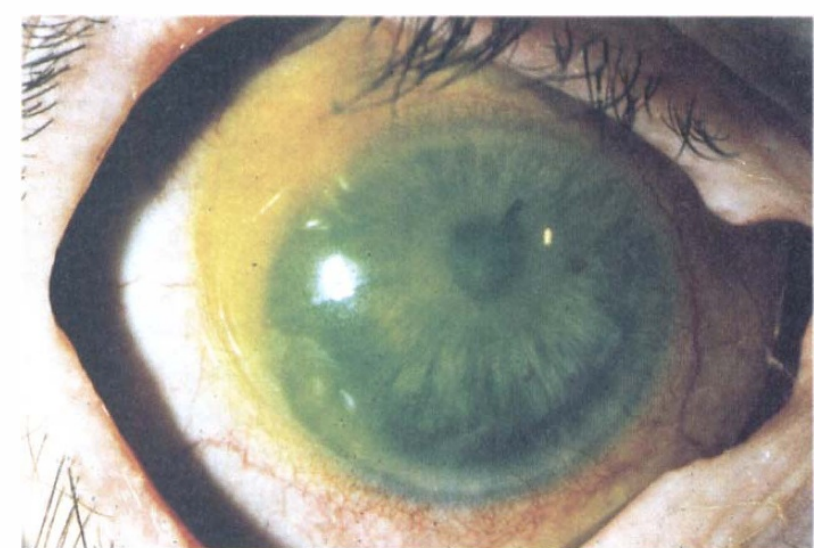

Fig. 2. Case 1. An RGP Scl CL in situ showing fluorescein coverage of the entire cornea.

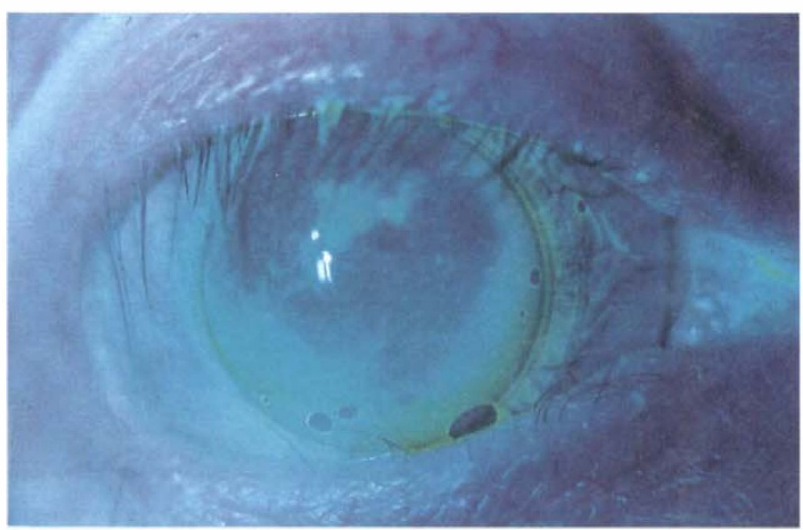

Fig. 4. Case 2. A second design, fitted with a corneal contact zone, gave a significant improvement in visual acuity.

\section{Rigid gas-permeable materials}

The introduction of rigid gas-permeable (RGP) materials ${ }^{4}$ has led to greater tolerance of Scl CLs because of increased corneal oxygenation ${ }^{5-8}$ and has simplified the fitting processes because sealed rather than fenestrated designs are possible in most cases. ${ }^{9-13}$

The introduction of RGP materials has enhanced the potential use for Scl CLs, especially for therapeutic applications, because they can be fitted to retain a satisfactorily oxygenated pre-corneal fluid reservoir. In particular, the improved oxygenation offers an increased potential for overnight wear. If insertion and removal is possible, they can be removed for cleaning during the waking hours and reinserted immediately, thereby providing daytime and overnight wear, but not uninterrupted continuous wear.

\section{Case studies}

A series of 7 index cases was selected to illustrate specific therapeutic management principles in a range of conditions with overnight Scl CL wear. Six of the cases described were assessed or reviewed in the last 3 years and were issued with RGP Scl CLs for overnight wear. One was a long-standing PMMA Scl CL wearer. All had undergone a successful scleral lens trial and were wearing the lens overnight at subsequent follow-up. At 


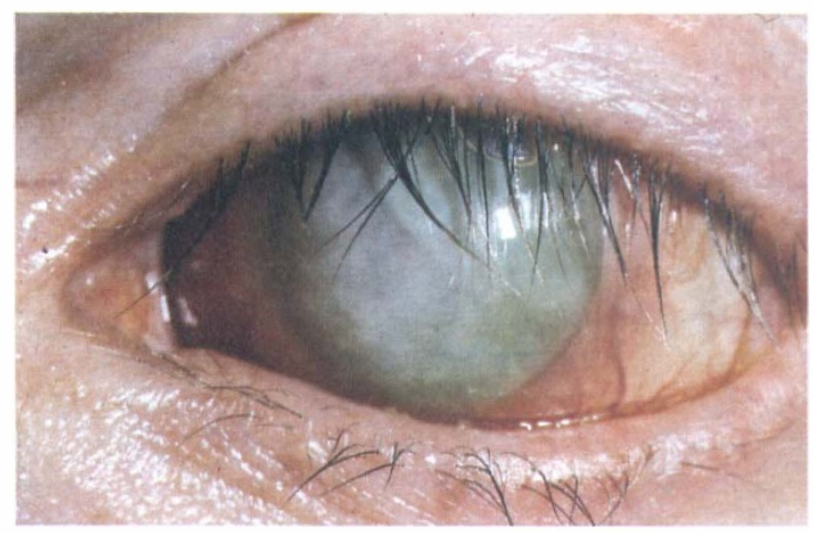

Fig. 5 Case 3. Radiation keratopathy and trichiasis. The left eye, showing trichiasis and corneal scarring, with an RGP Scl CL in situ.

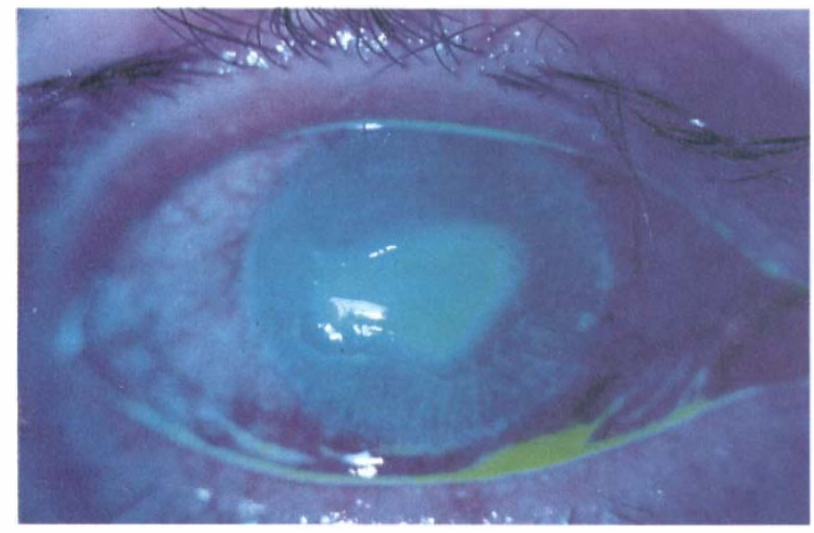

Fig. 7. Case 6. Lesion consequent to exposure and corneal anaesthesia following an acoustic neuroma. The extent of fluorescein staining is shown just prior to commencement of overnight wear.

the time of writing, the follow-up period ranged from 3 to 33 months for RGP Scl CLs. In the case of the single PMMA Scl CL wearer included in the study, the period was over 30 years. Patients who failed a Scl CL trial, or did not present at follow-up wearing overnight, were not included in this study.

Case 1: Illustrating overnight RGP Scl CL wear in the management of an immobile eye in order to control a persistent corneal epithelial erosion

Twenty years ago, a man then aged 48 years developed a perisistent right corneal erosion (Fig. 1) following multiple retinal detachment operations after a cataract. The eye movements and lid closure were greatly reduced following the repeated surgery, causing exposure with constant discomfort. A hydrogel bandage contact lens to alleviate the pain was worn on a continuous-wear basis for the next 17 years, and replaced every 3 months. Infections became an increasing problem in latter years, and there were periods when contact lens wear was not possible, during which the corneal erosions recurred. There was some neovascularisation of the inferior cornea, which was considered to be partly due to the recurrent infections and partly to 17 years of continuous soft bandage lens wear.

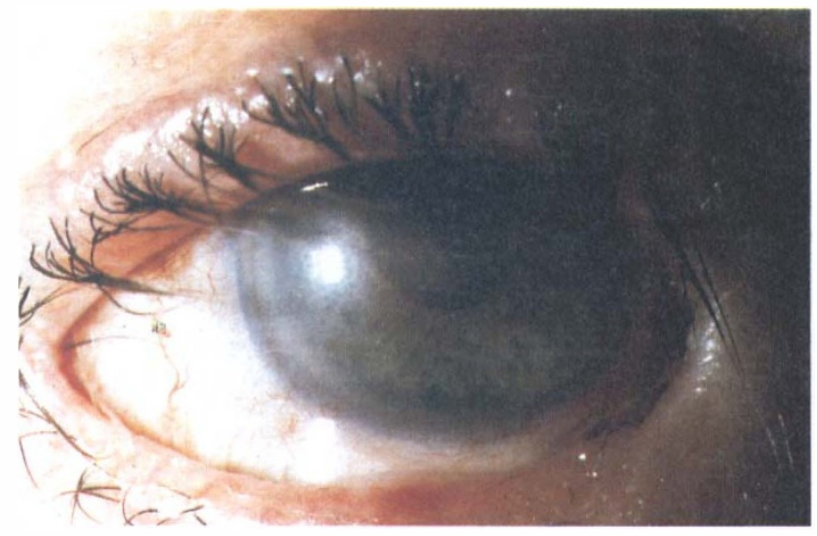

Fig. 6. Case 5. Corneal exposure following seventh nerve palsy, corrective lid surgery and partial tarsorrhaphy. The closure shown was the maximum possible, but the eye was quiet and comfortable with the RGP $S c l C L$ in situ.

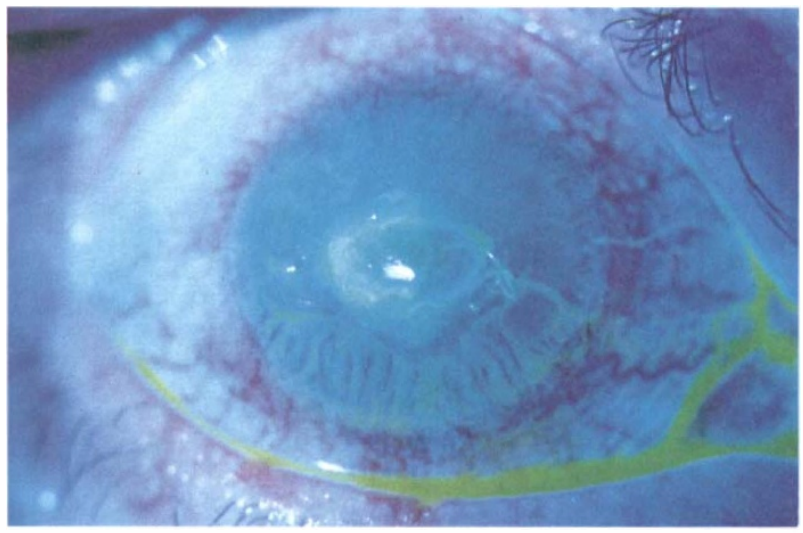

Fig. 8. Case 6. There is a reduction in the size of the central lesion after 2 weeks overnight wear of an RGP Scl CL.

In 1996 an RGP Scl CL was fitted with full corneal clearance (Fig. 2) in an attempt to alleviate the symptoms and reduce the frequency of infection. The patient was very reluctant to remove the lens for daily cleaning as recommended as he had only just managed to master the insertion and removal technique. In the event, the lens surface remained satisfactorily clean after 3 days of continuous wear.

At the most recent after-care consultation, which was 33 months after initiation of overnight wear, the patient was more comfortable, there had been no infections, and the corneal neovascularisation had not progressed since commencement of RGP Scl CL wear. The patient now removes, cleans and re-inserts the lens every $3-4$ days.

Case 2: Illustrating overnight RGP Scl CL wear to protect and to alleviate discomfort from trichisasis and to provide corneal hydration in the aftermath of Stevens Johnson disease

A female now aged 49 years developed Stevens Johnson disease at the age of 7 years. There was scarring of the lids with trichiasis, and corneal neovascularisation. A cataract developed which was extracted without insertion of an intraocular lens as its onset predated routine implantation. At age 20 years, she was issued with a PMMA Scl CL fitted from an eye impression for 
daytime use, giving a visual acuity of $6 / 24$. The left eye had been eviscerated following recurrent infected corneal perforations.

There was discomfort at night as well as during the waking hours, so an RGP Scl CL was tried for overnight wear in 1998. She was given a plano lens for use at night, so that the thickness could be made less than the high positive powered daytime lens. The lens for day wear gave improved visual acuity if fitted with a visual axis corneal contact zone (Fig. 3). The lens worn for overnight wear was made to alleviate the corneal contact (Fig. 4).

Eighteen months after initiation of overnight wear, the recorded visual acuity now varies between $6 / 12$ and $6 / 24$, although the patient reports a significant subjective improvement in her sight and a sustained reduction in the symptoms. This has been attributed to a reduction of corneal trauma from the trichiasis and improved corneal hydration.

Case 3: Illustrating overnight RGP Scl CL wear in the management of post-radiotherapy keratopathy and lid scarring with trichiasis

A male now aged 22 years was diagnosed with retinoblastoma aged 13 months. The right eye was enucleated, and radiotherapy applied to the left eye. In 1995 he developed a rhabdomyosarcoma of the left orbit. This was treated with further radiotherapy and melphalan with stem cell rescue.

Subsequently there has been severe radiation keratopathy and scarring of the upper eyelid with trichiasis and corneal exposure (Fig. 5). Initially this was managed with lubricants, which were insufficient to maintain comfort. A botulinum toxin-induced ptosis was used in the short term to improve the corneal surface, but was unacceptable as this was his only eye. An RGP Scl $\mathrm{CL}$ was fitted to protect the ocular surface from the trichiasis and to maintain corneal hydration.

A year after starting overnight lens wear the patient reports a subjective improvement in his visual clarity and ocular comfort although the recorded visual acuity remains at hand movements.

Case 4: Illustrating a rare example of long-term overnight PMMA Scl CL wear in the management of exposure keratitis secondary to multiple lid surgery for multiple congenital lid anomalies

A male now aged 53 years had a left congenital ptosis, with distichiasis and trichiasis. He underwent three operations during childhood to correct ptosis and lash malposition. This led to severe exposure of the cornea. Further surgery at the time was not recommended, so from 1958 the exposure was managed with an impression PMMA Scl CL for daytime and overnight wear, with removal for cleaning as necessary.

The situation remained unchanged for over 30 years, but there was significant corneal scarring and neovascularisation. This was attributable partly to the disease process and partly to contact lens wear, but was considered preferable to untreated exposure keratitis, especially as the discomfort was reduced.

Although previously not recommended, in 1989 a nasal septal cartilage graft to the left upper lid produced better lid closure and no contact lens was required for a period, but the pupil remained covered. A levator advancement elevated the upper lid, but led to recurrence of the exposure and discomfort, so Scl CL wear was resumed. This time, an RGP Scl CL with corneal clearance was tried. The patient preferred the PMMA Scl CL for day wear because the visual acuity was better, but used the RGP Scl CL at night to improve corneal oxygenation. This situation has remained unchanged for a further period of 10 years with a sustained visual acuity of 6/36.

Case 5: Illustrating overnight RGP Scl CL wear in the management of corneal exposure secondary to sixth and seventh cranial nerve palsy

A woman now aged 55 years suffered Weber's (brainstem CVA) syndrome in 1993, resulting in a left hemiplegia, right sixth and seventh cranial nerve palsies and secondary nystagmus in the left eye, resulting in reduced and confused vision. There was a poor Bell's phenomenon, with lagophthalmos and exposure of the right cornea. Conventional lubricants and lid taping at night proved to be insufficient and a lateral tarsorrhaphy was performed.

After 2 years, the patient was keen to improve the vision of the right eye, so the tarsorrhaphy was opened, but this led to corneal exposure with consequent discomfort. A silicone rubber bandage contact lens partly reduced the symptoms but did not maximise the visual acuity. There was also a rapid degradation of the lens surface. An RGP Scl CL fitting was initiated for use on a daily and overnight wearing regime (Fig. 6). The patient's husband easily learned the handling skills necessary for daily removal, cleaning and replacement of the lens.

After 1 year, the patient reports that the comfort is better than all the options previously tried, and does not require lubricants. The Scl CL gives a visual acuity of $6 / 12$ and enables better suppression of the nystagmic left eye.

\section{Case 6: Illustrating overnight RGP Scl CL wear in an eye rendered anaesthetic and exposed following surgery for an acoustic neuroma}

In October 1998, a man aged 58 years suffered an acoustic neuroma which was surgically treated, but left residual fifth and seventh cranial nerve palsies. There was exposure and anaesthesia of the right cornea. A $5 \mathrm{~mm}$ central corneal defect developed (Fig. 7) and was persistent in spite of a regime of lubricants and overnight lid-taping, reducing the acuity to hand movements. 
Lateral tarsorrhaphy and the insertion of a gold weight into the upper lid had been offered but was declined. An RGP Scl CL was fitted in February 1999, initially for daytime wear, but switching to overnight wear after an adaptation period. The epithelial defect reduced in size (Fig. 8), and after 2 months had healed. The patient continued to wear the lens for a period of 9 months with assistance from his wife for twice-daily cleaning. The patient has subsequently died, but the lens had a valuable therapeutic use for the last year of his life.

Case 7: Illustrating overnight but not daytime wear of an RGP Scl CL in the management of corneal exposure consequent to poor night closure following multiple lid surgery after childhood trauma

A female now aged 37 years had sustained a facial laceration that caused avulsion of the left upper and lower lids at age 3 years. She had multiple lid surgery spanning 34 years but a left exotropia and amblyopia remained. The cornea sustained repeated erosions, which further reduced the vision to 3/60. The surgical repair and subsequent procedures were essentially successful, but left an incomplete night closure leading to discomfort and requiring lid-taping to allow uninterrupted sleep. There was no significant discomfort during the day. In 1999, she was issued with a sealed RGP Scl CL as an alternative to further surgery for night use only.

After 3 months, she reported almost complete resolution of the discomfort with no need for lid-taping. The neutralisation of the disrupted corneal surface gave a visual acuity of $6 / 24$ but as the right vision was $6 / 5$, she did not find this unilateral improvement a worthwhile advantage.

\section{Discussion}

The cases reported in the current paper demonstrate the variety of conditions for which Scl CLs may have a therapeutic application when worn overnight. In all cases the patients were issued with RGP Scl CL for overnight wear. One patient, who was a long-standing PMMA Scl CL wearer, was issued with an RGP Scl CL for overnight wear but retained his PMMA Scl CL for daytime wear as this gave better vision.

In every case, the Scl CLs were removed and cleaned regularly, in contrast to the concept of continuous wear hydrogel or silicone lens wear in which case the lens may be left in situ until such time it has to be replaced. In some cases, the outcome was more successful than with hydrogel or silicone rubber lenses because the exposure symptoms were more effectively dealt with; in other examples, there was better protection from trichiasis. If the eye was sighted, the acuity with a Scl CL compared favourably with that achieved with hydrogel or silicone rubber lenses in all cases described in this study.

The authors do not suggest that RGP SCI CLs should be the first choice in cases where therapeutic contact lenses may be applied. However, as most of the patients in this study were switched from hydrogel or silicone rubber lenses to RGP Scl CLs, it is clear that with judicious selection, they have a worthwhile role.

\section{Conclusion}

The cases presented show that Scl CLs constitute a useful therapeutic option for some patients with severe corneal or anterior ocular surface disease. The use of highly gaspermeable materials permits overnight wear Scl CLs to be considered as an alternative to hydrogel or silicone rubber therapeutic lenses in managing complicated anterior eye conditions for which other methods are either unsuitable or less effective.

\section{References}

1. Smiddy WE, Hamburg TR, Kracher GP. Therapeutic contact lenses. Ophthalmology 1990;97:291-5.

2. Liesegang TJ. Contact lens-related microbial keratitis. I. Epidemiology. Cornea 1997;16:125-31.

3. Tan DTH, Pullum KW, Buckley RJ. Medical applications of scleral contact lenses. A retrospective analysis of 343 cases. Cornea 1995;14:121 9.

4. Ezekiel D. Gas permeable haptic lenses. J Br Contact Lens Assoc 1983;6:158-61.

5. Ruben CM, Benjamin WJ. Scleral contact lenses: preliminary report on oxygen-permeable materials. Contact Lens 1985;13:5-10.

6. Pullum KW, Hobley AJ, Parker JH. Dallos award lecture. Part II: Hypoxic corneal changes following sealed gas permeable impression scleral lens wear. J Br Contact Lens Assoc 1990;13:83-7.

7. Mountford J, Carkeet N, Carney L. Corneal thickness changes during scleral lens wear: effect of gas permeability. ICLC 1994;21:19-21.

8. Pullum KW, Stapleton FJ. Scleral lens induced corneal swelling: what is the effect of varying Dk and lens thickness? CLAO J 1997;23:259-63.

9. Visser R. Een nieuwe toekomst hoogzuurtofdooreatende scleralenzen bij verschillende pathologie. Ned Tijdschr Optom Contactol 1990;3:10-4.

10. Schein OD, Rosenthal P, Ducharme C. A gas-permeable scleral contact lens for visual rehabilitation. Am J Ophthalmol 1990;109:318-22.

11. Kok JHC, Visser R. Treatment of ocular surface disorders and dry eyes with high gas-permeable scleral lenses. Cornea 1992;11:518-22.

12. Tan DTH, Pullum KW, Buckley RJ. Medical applications of scleral contact lenses. II. Gas-permeable scleral contact lenses. Cornea 1995;14:130-7.

13. Pullum KW, Buckley RJ. A study of 530 patients referred for rigid gas permeable scleral contact lens assessment. Cornea 1997;16:612-22. 